\title{
Uso diário e semanal de sulfato ferroso no tratamento de anemia em mulheres no período reprodutivo
}

\author{
Use of daily and weekly ferrous sulfate \\ to treat anemic childbearing-age women
}

Maria do Carmo dos Santos Lopes 1

Luiz Oscar Cardoso Ferreira 2

Malaquias Batista Filho 3

\footnotetext{
1 Departamento de Enfermagem, Saúde Pública e Ciências do Comportamento, Faculdade de Enfermagem Nossa Senhora das Graças, Universidade de Pernambuco. Rua Arnóbio Marques 310, Recife, PE, 50100-130, Brasil. 2 Departamento de Medicina Social, Faculdade de Ciências Médicas, Universidade de Pernambuco. Rua Arnóbio Marques 310, Recife, PE, 50100-130, Brasil. 3 Departamento de Nutrição, Universidade Federal de Pernambuco. Rua dos Coelhos 300 Recife, PE, 50070-550, Brasil.
}

\begin{abstract}
A blind randomized trial was conducted in a low-income community in the city of Recife, Pernambuco, Brazil, with 193 anemic $(H b<12 \mathrm{mg} / \mathrm{dl}$ ) and "menstruating" women (age range: 15-45 years) to compare daily and weekly doses of ferrous sulfate (60 mg elemental iron) and treatment compliance. Blood samples were taken from 484 women to determine hemoglobin levels before and after the trial and mean corpuscular volume (MCV) at end of treatment. After 12 weeks' follow-up, 150 women completed the trial, 79 on the alternative weekly regimen and 71 on the conventional daily regimen. Mean corpuscular hemoglobin concentrations (MCHC) prior to treatment were $10.52 \mathrm{~g} / \mathrm{dl}(\mathrm{DP}=1.13)$ and $10.72 \mathrm{~g} / \mathrm{dl}(\mathrm{DP}=0.92)$, respectively, for the alternative and conventional regimens. After the intervention they were $11.83 \mathrm{~g} / \mathrm{dl}(\mathrm{DP}=0.97)$ for the weekly regimen and $11.62 \mathrm{~g} / \mathrm{dl}(\mathrm{DP}=1.39)$ for the daily one. The alternative regimen was better accepted than the conventional one. There was no significant difference $(p=0.22)$ between differences in the mean values of the two regimens, although the cure rate was higher after the alternative treatment. The study concluded that the weekly regimen was no less effective than daily treatment.
\end{abstract}

Key words Anemia; Iron; Ferrous Sulfate; Women's Health

Resumo Mediante um ensaio terapêutico randomizado e cego em 193 mulheres (15-45 anos de idade) anêmicas ( $H b<12 \mathrm{mg} / \mathrm{dl}$ ) e "menstruantes", comparou-se a ação e a adesão ao tratamento com sulfato ferroso (60 mg de Fe elementar), administrado em doses diárias e semanais, em uma comunidade de baixa renda do Recife-PE. Após 12 semanas de tratamento, 150 mulheres chegaram ao final do ensaio, sendo 79 do esquema semanal e 71 do diário. As médias de Hb antes do tratamento foram, respectivamente, 10,52 g/dl (DP =1,13) e 10,72 g/dl (DP =0,92) para o esquema alternativo e convencional. Após a intervenção, as médias de Hb alcançaram 11,83 g/dl $(D P=0,97)$ no esquema semanal e 11,62 g/dl $(D P=1,39)$ no diário. Não houve significância estatística $(p=0,22)$ entre as diferenças de médias nos dois esquemas terapêuticos, embora o percentual de cura $(48,1 \%)$ tenha sido maior no tratamento semanal, quando comparado ao diário (36,6\%). Conclui-se que o esquema de tratamento semanal teve a mesma eficácia do diário. A aceitação ao tratamento no primeiro mês foi melhor no esquema semanal, não havendo, entretanto, diferenças nos dois meses subseqüentes.

Palavras-chave Anemia; Ferro; Sulfato Ferroso; Saúde da Mulher 


\section{Introdução}

No quadro epidemiológico dos problemas nutricionais em escala mundial, as anemias configuram, por sua magnitude, a situação carencial de maior prevalência, atingindo, segundo Viteri et al. (1993), cerca de 2,15 bilhões de pessoas, o que eqüivale a aproximadamente $35 \%$ da população humana.

As crianças menores de cinco anos, as mulheres na idade fértil e, sobretudo, no período gestacional, constituem, em virtude de fatores biológicos, os segmentos populacionais mais expostos ao problema. Além de sua elevada magnitude, as anemias têm marcantes implicações no processo saúde/doença, interagindo com outros agravos carenciais e não-carenciais, contribuindo, assim, para elevar as estatísticas de morbi-mortalidade (Oliveira, 1990). Reduzem, ainda, a capacidade de aprendizagem e o rendimento do trabalho físico (Finch \& Cook, 1984).

O papel das condições sócio-econômicas na etiologia das doenças carenciais, e particularmente na anemia nutricional, está bem estabelecido (Martins et al., 1987; Batista Filho \& Ferreira, 1996). Um estudo realizado pela Organização Mundial da Saúde, em 1968 sobre a prevalência da anemia por deficiência de ferro em mulheres que menstruavam e sua relação com o nível sócio-econômico, mostrou que, enquanto nos países desenvolvidos a anemia por carência de ferro teria uma ocorrência de $10 \%$, nos países em desenvolvimento alcançava $50 \%$ (Finch \& Cook, 1984). Assim, embora afetando principalmente as populações dos países pobres, o problema também se difunde como ocorrência endêmica nas nações ricas, estimando-se que, na Europa, 27 milhões de habitantes apresentam esse tipo de deficiência (Viteri et al., 1993).

No Brasil, como em outros países, as informações sobre a prevalência da anemia são ainda escassas. Dados disponíveis a partir de estudos realizados em algumas regiões do país, nos últimos 30 anos, indicam que a prevalência de anemia ferropriva situa-se entre $22 \%$ a 45\% (PAHO/WHO, 1994), resultados compatíveis com estudos realizados em Pernambuco por Arruda (1990, 1997) e Pereira (1997).

A perda básica de ferro em mulheres que não menstruam é de $0,6 \mathrm{mg}$ a $0,9 \mathrm{mg} \mathrm{Fe} /$ dia (Rybo, 1970). Durante a menstruação, esse valor se eleva para 2 mg ou mais (Rapaport, 1990; Dallman, 1991). É muito variável a quantidade de sangue eliminada durante cada menstruação, perdendo-se em média $25 \mathrm{ml}$ a $30 \mathrm{ml}$ de sangue (Rybo, 1970; Hercberg \& Galan, 1992).
Calcula-se uma redução de $50 \%$ a $60 \%$ de perda sangüínea, nos períodos menstruais, em mulheres que fazem uso de contraceptivos orais contendo estrógeno (Rybo, 1970; Hilmann, 1991). Durante o período reprodutivo, as mulheres necessitam de cerca de 1,4 mg/Fe/dia. Entretanto, a dieta, em muitas situações, não fornece ferro suficiente para as necessidades na vida adulta, principalmente, por causa das perdas sangüíneas menstruais e das gestações (Rapaport, 1990; Hercberg \& Galan, 1992). Isso reforça a afirmação de alguns autores de que, com certa freqüência, as mulheres iniciam a gestação com reservas insuficientes do mineral, tornando-se mais propensas à anemia (Martins et al., 1987; Dallman, 1991).

Apesar de ser reconhecida em estudos antigos de nosografia humana, somente na década atual a importância do problema foi formalmente validada, com a declaração dos chefes de estados, reunidos sob o patrocínio das Nações Unidas, incluindo-se o combate às anemias no plano decenal de metas proposto para o ano 2000 (Encontro Mundial de Cúpula pela Criança, 1990). Restringindo-se às mulheres no período reprodutivo, a diminuição de um terço da prevalência da anemia constitui o mais modesto dos compromissos internacionais assumidos em relação aos problemas de nutrição humana para o referido decênio (Batista Filho \& Ferreira, 1996).

A possibilidade técnica, científica e cultural de que estas metas possam ser superadas com a adoção de tratamentos alternativos, como a utilização de doses semanais de sais de ferro, torna oportuna e pertinente a focalização destes objetivos, mediante ensaios clínicos devidamente controlados. Isto poderia proporcionar a possibilidade de melhorar consideravelmente as perspectivas de cumprimento e, até, de superação das metas previstas para o ano 2000 (Batista Filho \& Ferreira, 1996).

Segundo Stekel (1984), o ideal seria que a prevenção das anemias e outras deficiências nutricionais pudesse ser conseguida por meio de uma boa dieta. Infelizmente, muitas vezes isso não é possível, uma vez que a quantidade total de ferro dietético e sua biodisponibilidade não atendem às necessidades estabelecidas.

A prevenção e a terapêutica para a anemia nutricional ferropriva, a partir da utilização de compostos de ferro, ainda hoje plenamente válida, constitui um dos mais antigos recursos de tratamento (Batista Filho \& Ferreira, 1996). O sulfato ferroso administrado por via oral é o mais recomendado, devido ao seu baixo custo e alta biodisponibilidade (Adish \& Esrey, 1996). 
A absorção do ferro se processa pelas células da mucosa duodeno-jejunal, que dispõem de mecanismo de controle para que sua assimilação não se torne excessiva. Este recurso homeostático também opera em sentido contrário, aumentando a eficiência de absorção nos casos de escassez ou de necessidades aumentadas (Hillman, 1991).

Os sais de ferro são, geralmente, bem tolerados (Oliveira, 1990), podendo, no entanto, produzir efeitos colaterais, como pirose, náuseas, vômitos, diarréia, constipação intestinal e cólicas (Stekel, 1984; Oliveira, 1990), ocorrendo em $15 \%$ a $20 \%$ dos pacientes em tratamento diário com ferro oral (Cardoso \& Penteado, 1994). Muitas pessoas são pouco motivadas a tratar a anemia com sais de ferro, especialmente quando se administra doses altas, por causa da longa duração do tratamento e dos efeitos indesejáveis (Schultink et al., 1995; Batista Filho \& Ferreira, 1996), contribuindo, assim, para o insucesso dessa medida. É um sério problema a ser contornado.

Viteri et al. (1993) propõem a utilização de doses semanais em substituição às doses diárias, partindo do princípio de que a mucosa intestinal bloqueia a absorção do ferro medicamentoso quando administrado repetidamente. Contornada a barreira do bloqueio epitelial, cujas células se renovam a cada oitenta horas (Lipkin et al., 1963), o tratamento seria simplificado, os efeitos colaterais reduzidos, o custo baixaria, esperando-se maior eficiência e efetividade nas intervenções de ampla cobertura. Schultink et al. (1995), utilizando em crianças doses de $30 \mathrm{mg}$ de ferro elementar diariamente e em outro grupo duas vezes por semana, observaram que não ocorreram diferenças estatisticamente significativas entre os dois esquemas de tratamento.

O emprego de fórmulas medicamentosas mais complexas também parece não apresentar vantagens terapêuticas em relação à medicação apenas com sulfato ferroso. Um estudo realizado no Instituto Materno Infantil de Pernambuco com 177 gestantes anêmicas demonstrou que altas ou baixas doses de ferro, usadas de forma isolada ou em combinação com folato e vitamina $\mathrm{B}_{12}$, não alteraram os resultados parciais e finais da intervenção, demonstrando que a eficácia terapêutica dependeria mais da duração do tratamento do que da dosagem e combinação de nutrientes hematínicos (Marques, 1992).

Portanto, a hipótese de que a absorção de ferro no organismo seria igual ou maior quando se administra sulfato ferroso uma vez por semana e que os efeitos colaterais seriam me- nores amplia as perspectivas de que, com os mesmos ou com menos recursos, seja possível maximizar o atendimento aos grupos de elevado risco, com maior aceitação da terapêutica e, conseqüentemente, melhoria dos resultados finais. São estes os objetivos do presente estudo.

\section{Procedimentos metodológicos}

O ensaio foi realizado na comunidade de Santo Amaro, na cidade do Recife, Nordeste do Brasil. Quase um terço da população da cidade vive em favelas, onde $50 \%$ das famílias têm renda mensal abaixo de 100 dólares, 70\% dos domicílios não têm esgotos sanitários, $20 \%$ não dispõem de abastecimento de água adequado e 15\% da população é analfabeta (IBGE, 1992).

A comunidade favelada de Santo Amaro tem cerca de 17 mil habitantes (Prefeitura da Cidade do Recife, 1993). A maioria das ruas da comunidade não tem calçamento e algumas estão situadas sobre mangues, constituídas por casas de alvenaria, taipa e barracos de tábua, zinco e papelão.

O estudo realizado foi um ensaio clínico randomizado cego. Assim, o responsável pela avaliação hematológica não conhecia as hipóteses, objetivos e tipos de tratamento a que foram submetidos os participantes da pesquisa.

A população estudada foi constituída por mulheres em idade reprodutiva, na faixa etária de 15 a 45 anos, não-gestantes, que menstruavam regularmente ("menstruantes") e com níveis de hemoglobina entre 7,5 g/dl e 11,9 g/dl. Foram excluídas as mulheres que referiram estar tomando ou tomaram sulfato ferroso nos últimos trinta dias, as portadoras de doenças hematológicas e renais, bem como aquelas que, por livre deliberação, não concordaram em participar da experiência.

Para o cálculo do tamanho da amostra, utilizou-se a fórmula de Kirkwood (1988), baseada na diferença de médias $(\alpha=0,05 \mathrm{~g} / \mathrm{dl} ; 1-\beta=$ $0,8 \mathrm{~g} / \mathrm{dl} ; \delta=0,5 \mathrm{~g} / \mathrm{dl}$ ). Obteve-se, assim, um valor de 81 mulheres por grupo, ou seja, 162 para os dois grupos. Acrescentando $20 \%$ como margem de segurança, estimou-se que 200 mulheres seriam suficientes para o início do estudo.

A variável explicativa (independente) foi o tipo de tratamento, diário e semanal, com sulfato ferroso heptahidratado $\left(\mathrm{F}_{\mathrm{e}} \mathrm{SO}_{4} .7 \mathrm{H}_{2} \mathrm{O}\right)$, contendo $60 \mathrm{mg}$ de ferro elementar, em forma de comprimidos revestidos. As variáveis respostas (dependentes) foram os níveis de hemoglobina antes do tratamento e os níveis de hemoglobina e o volume corpuscular médio (VCM) após o uso da medicação e, por fim, a adesão ao tratamento. 
Para avaliar a concentração de hemoglobina no início do estudo, utilizou-se amostras de sangue, colhidas através de punção digital, procedendo-se à leitura pelo método de Drabkin modificado (Kjeldsberg, 1993), com o hemoglobinômetro portátil, tipo CELM. É um método simples, rápido e de baixo custo, prestandose como um excelente screening. Na segunda etapa, após 12 semanas de tratamento, além da hemoglobina, foram estimados os valores do VCM, objetivando uma maior precisão diagnóstica por contador eletrônico de células, o Coulter T. 890.

A adesão aos tipos de tratamento foi classificada como ótima, quando seguida a recomendação terapêutica exatamente como prescrita, e satisfatória, quando a prescrição deixou de ser cumprida fielmente, sem, no entanto, comprometer o tratamento. A condição insatisfatória foi atribuída aos casos em que, no tratamento semanal, as pesquisadas deixaram de tomar um comprimido de sulfato ferroso, e no tratamento diário, mais de $10 \%$ da medicação, ou seja, mais de três comprimidos.

Outras variáveis consideradas importantes para o estudo foram a idade, nível de instrução, índice de massa corporal (IMC) e condições econômicas, representadas pela renda per capita familiar e pelos bens de consumo duráveis.

A alocação dos esquemas de tratamentos foi efetuada de forma aleatória, mediante sorteio simples. Um grupo recebeu o tratamento que se queria testar, considerado alternativo ou experimental, com sulfato ferroso em doses semanais, e o outro grupo recebeu a terapêutica habitual, esquema convencional, com sulfato ferroso em doses diárias.

Após recrutamento, realização dos exames e processo de exclusão, foram selecionadas 193 mulheres anêmicas, das quais 96 receberam tratamento com dose diária e 97 tratamento com dose semanal. Ao final das 12 semanas, em razão das perdas, permaneceram 150 mulheres, sendo 79 com o tratamento alternativo em doses semanais e 71 com o tratamento convencional de doses diárias.

O estudo foi realizado no período de fevereiro a setembro, sendo o tratamento administrado entre junho e agosto de 1996. As mulheres que apresentaram níveis de hemoglobina entre 7,5 g/dl e 11,9 g/dl responderam a um questionário e receberam o sulfato ferroso, formando os grupos de tratamento alternativo e de tratamento convencional. A cada quatro semanas foi aplicado questionário e distribuída a medicação. Após o término do ensaio, os resultados foram divulgados para as participantes, e as que continuaram anêmicas foram referen- ciadas para o clínico do posto de saúde da comunidade.

Amostras de comprimidos de sulfato ferroso foram submetidas a análises farmacológicas no Laboratório Farmacêutico do Estado de Pernambuco (LAFEPE) para verificação dos teores de ferro indicados nas embalagens. Enviou-se amostras de sangue venoso, em duplicata, para se averiguar a reprodutibilidade do método utilizado.

\section{Resultados}

Por definição prévia, a idade da população variou de 15 a 45 anos, com a média de 27,4 (DP = 8,0 ) anos.

Em relação ao nível de instrução, observouse que as mulheres cursaram em média 5,4 (DP $=3,0$ ) anos de estudo, sendo 6,7\% analfabetas. Quase a metade, $42 \%$, freqüentou a primeira fase do ensino fundamental, e apenas uma mulher freqüentou curso superior.

A média da renda mensal monetária per capita foi de $\mathrm{R} \$ 46,18$ (DP = 46,17). Chama a atenção a elevada dispersão dos valores em torno da média. Observou-se que $28,2 \%$ das mulheres moravam em domicílios que tinham renda mensal per capita abaixo da linha de pobreza, ou seja, menor que R\$25,00. Apenas $8 \%$ das famílias auferiam rendimentos iguais ou superiores a $\mathrm{R} \$ 100,00$ mensais, por pessoa. A presença de televisão e geladeira funcionando foi detectada em $91,7 \%$ e $69,9 \%$ das casas, respectivamente.

Segundo o índice de massa corporal (IMC), $9,8 \%$ das mulheres foram consideradas como casos de baixo peso, $55,5 \%$ estavam com o peso normal, enquanto que $34,7 \%$ apresentavam peso acima do normal.

Por definição metodológica, o experimento foi realizado apenas em mulheres anêmicas, com níveis de hemoglobina entre 7,5 g/dl e 11,9 $\mathrm{g} / \mathrm{dl}$ e observada uma média de 10,6 g/dl (DP = 1,02 ), sendo que $6,7 \%$ apresentaram hemoglobina abaixo de 9,0 g/dl, 45,6\% tinham teores compreendidos entre 9,0 g/ dl e 10,9 g/ dl e 47,7\% entre 11,0 g/dl e 11,9 g/dl de hemoglobina.

A distribuição das características da amostra estudada em relação aos dois tratamentos administrados (Tabela 1) mostra que os grupos eram estatisticamente comparáveis, ao nível de 5\%, em relação a idade, instrução, posse de bens duráveis, índice de massa corporal e, finalmente, médias de hemoglobina.

Os resultados dos dois tratamentos achamse sumariados na Tabela 2 , considerando as diversas características da variável resposta. 
Características iniciais dos dois grupos de mulheres segundo o esquema de tratamento. Santo Amaro, Recife, 1996.

\begin{tabular}{|c|c|c|c|c|}
\hline \multirow[t]{2}{*}{ Características } & \multicolumn{2}{|c|}{ Tratamento* } & \multirow[t]{2}{*}{ Estatística } & \multirow[t]{2}{*}{ "p" } \\
\hline & Alternativo & Convencional & & \\
\hline Idade (anos) $x \pm D P$ & $26,6 \pm 7,7$ & $28,2 \pm 8,3$ & $H=1,731$ & 0,1883 \\
\hline Instrução (anos) $x \pm D P$ & $5,3 \pm 2,9$ & $5,5 \pm 3,2$ & $H=0,001$ & 0,9844 \\
\hline Renda $(R \$) x \pm D P$ & $36,67 \pm 23,95$ & $56,78 \pm 60,72$ & $\mathrm{H}=4,290$ & 0,0383 \\
\hline Posse de TV \% & 91,7 & 91,8 & $\chi^{2}=0,001$ & 0,9827 \\
\hline Posse de Geladeira \% & 71,9 & 68,0 & $\chi^{2}=0,340$ & 0,5613 \\
\hline$I M C x \pm D P$ & $23,5 \pm 4,8$ & $23,9 \pm 4,7$ & $H=0,566$ & 0,4517 \\
\hline $\mathrm{Hb}(\mathrm{g} / \mathrm{dl}) \times \pm \mathrm{DP}$ & $10,56 \pm 1,07$ & $10,62 \pm 0,97$ & $H=0,018$ & 0,8940 \\
\hline
\end{tabular}

* Tratamento alternativo - doses semanais. Tratamento convencional - doses diárias.

Resultados dos esquemas de tratamento alternativo e convencional. Santo Amaro, Recife, 1996.

\begin{tabular}{lll}
\hline Características & & Tratamento \\
& Alternativo & Convencional \\
\hline Número de participantes & 79 & 71 \\
Média e DP de Hb antes & $10,52 \pm 1,13$ & $10,72 \pm 0,92$ \\
Média e DP de $\mathrm{Hb}$ depois & $11,83 \pm 0,97$ & $11,62 \pm 1,18$ \\
$\%$ de cura $(\mathrm{Hb} \geq 12 \mathrm{~g} / \mathrm{dl})$ & 48,1 & 36,6 \\
$\%$ de anemia microcítica & 22,0 & 17,8 \\
\hline
\end{tabular}

O teste de comparação de médias mostrou que não houve diferença estatística significativa, quando comparados os resultados finais dos dois tratamentos testados, apesar do percentual de cura mostrar-se maior no tratamento alternativo de doses semanais.

A adesão aos dois esquemas de tratamento foi acompanhada em três momentos da coleta dos dados (após o primeiro, o segundo e o terceiro mês), conforme mostra a Tabela 3. Observou-se que no tratamento alternativo (doses semanais), a adesão foi mais elevada nos três momentos estudados. O teste do qui quadrado mostrou que as diferenças observadas foram estatisticamente significativas após um mês de seguimento.

Durante o período de tratamento ocorreram algumas perdas, conforme observado no fluxograma (Figura 1), mostrando a evasão de oito casos após as quatro primeiras semanas, cinco após as quatro semanas seguintes e, finalmente, 30 nas quatro últimas semanas, perfazendo um total de 43 perdas durante as 12 semanas do tratamento.
Com a finalidade de esclarecer se estas perdas foram diferentes tanto em relação ao tratamento administrado quanto às características da amostra estudada, com possível interferência nos resultados, foi elaborado a Tabela 4 , resumindo as principais características das perdas em relação à composição da amostra original do estudo. Verificou-se que as perdas não afetaram a composição final do grupo estudado em relação aos seus atributos iniciais.

\section{Discussão}

Tratando-se de um ensaio terapêutico, obviamente o padrão da prevalência em si não assume maior interesse, na medida em que define um indicador mais aplicado às abordagens epidemiológicas. No entanto, convém ressaltar a elevada ocorrência das anemias $(42,1 \%)$ na população estudada, superior ao que se poderia esperar de estudos prévios (Salzano et al., 1980; Arruda, 1990, 1997) e equivalente aos resultados descritos pelas Nações 
Tabela 3

Adesão aos esquemas de tratamento em três momentos do estudo. Santo Amaro, Recife, 1996

\begin{tabular}{|c|c|c|c|c|c|}
\hline \multirow[t]{3}{*}{ Momento (meses) } & \multicolumn{4}{|c|}{ Aderência } & \multirow[t]{3}{*}{ Total } \\
\hline & \multicolumn{2}{|c|}{ Satisfatória } & \multicolumn{2}{|c|}{ Ótima } & \\
\hline & $\mathrm{n}$ & $\%$ & $\mathrm{n}$ & $\%$ & \\
\hline \multicolumn{6}{|l|}{ 1으ês } \\
\hline Tratamento convencional & 21 & 22,4 & 73 & 77,6 & 94 \\
\hline Tratamento alternativo & 8 & 8,7 & 83 & 91,3 & 91 \\
\hline Total & 29 & & 156 & & 185 \\
\hline Estatística & $\chi^{2}=6,42$ & $p=0,01$ & & & \\
\hline \multicolumn{6}{|l|}{ 2o Mês } \\
\hline Tratamento convencional & 16 & 17,8 & 74 & 83,2 & 90 \\
\hline Tratamento alternativo & 9 & 10,0 & 81 & 90,0 & 90 \\
\hline Total & 25 & & 155 & & 180 \\
\hline Estatística & $\chi^{2}=2,28$ & $p=0,13$ & & & \\
\hline \multicolumn{6}{|l|}{ 3o Mês } \\
\hline Tratamento convencional & 13 & 16,5 & 66 & 83,5 & 79 \\
\hline Tratamento alternativo & 7 & 9,8 & 64 & 90,2 & 71 \\
\hline Total & 20 & & 130 & & 150 \\
\hline Estatística & $\chi^{2}=1,41$ & $p=0,24$ & & & \\
\hline
\end{tabular}

Figura 1

Fluxograma do estudo.

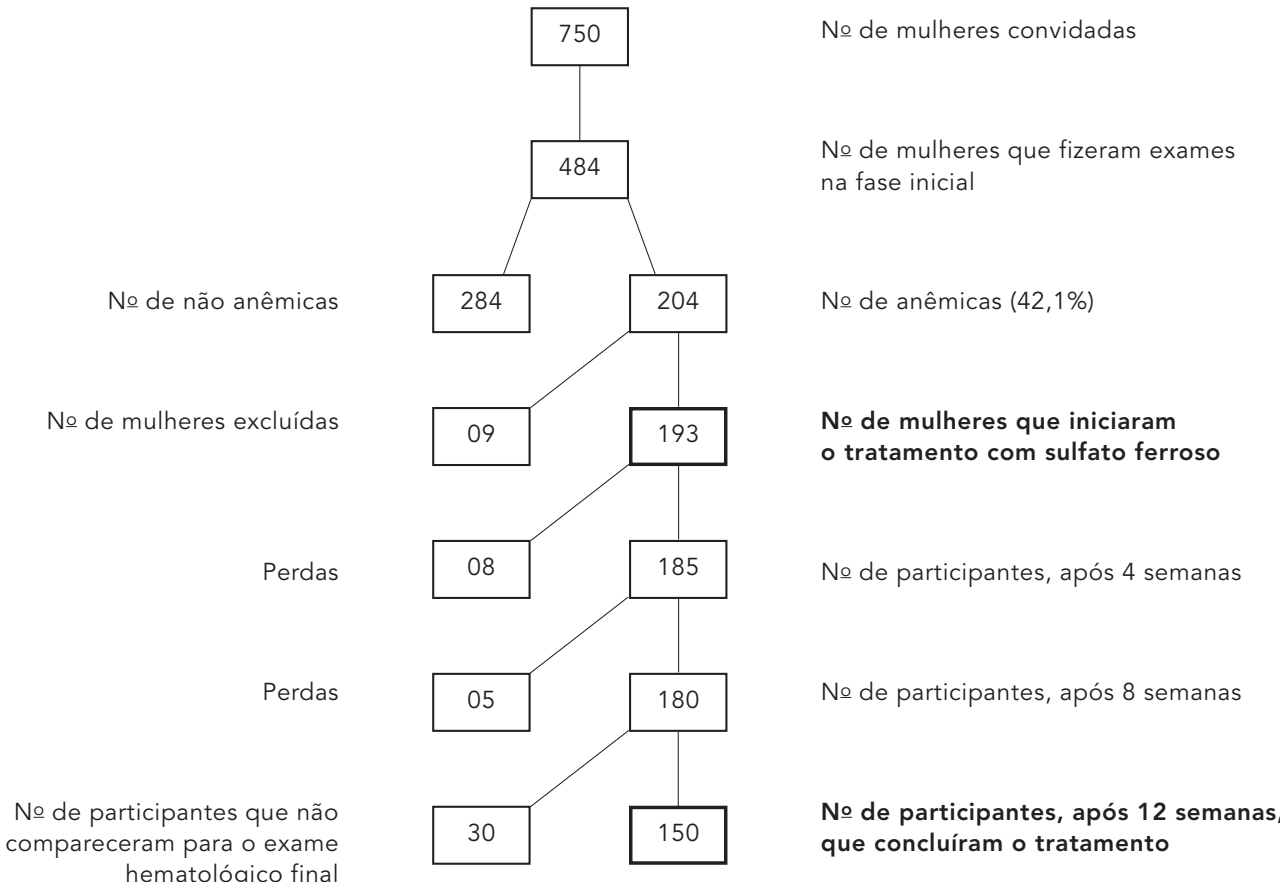


Características da amostra do estudo e das perdas ocorridas. Santo Amaro, Recife, 1996.

\begin{tabular}{|c|c|c|c|c|}
\hline Características & Amostra & Perdas & Estatística & "p" \\
\hline Idade (anos) $x \pm D P$ & $27,4 \pm 8,0$ & $25,3 \pm 6,8$ & $H=3,276$ & 0,0703 \\
\hline Instrução (anos) $x \pm D P$ & $5,4 \pm 3,0$ & $4,9 \pm 3,0$ & $H=1,358$ & 0,2438 \\
\hline Renda $(\mathrm{R} \$) \times \pm D P$ & $46,2 \pm 46,2$ & $44,1 \pm 41,6$ & $H=0,256$ & 0,6145 \\
\hline Posse de TV \% & 91,7 & 95,3 & $\chi^{2}=0,960$ & 0,3262 \\
\hline Posse de Geladeira \% & 69,9 & 60,5 & $\chi^{2}=2,370$ & 0,1239 \\
\hline$I M C \times \pm D P$ & $23,7 \pm 4,8$ & $22,2 \pm 3,8$ & $H=5,387$ & 0,0202 \\
\hline $\mathrm{Hb}(\mathrm{g} / \mathrm{dl}) \mathrm{x} \pm \mathrm{DP}$ & $10,6 \pm 1,0$ & $10,5 \pm 1,0$ & $H=0,758$ & 0,3838 \\
\hline
\end{tabular}

Unidas para o continente africano (Naciones Unidas, 1987).

Esta particularidade, além de ressaltar a magnitude do problema em um ecossistema urbano singularmente desfavorável (área de favela), muito comum no Brasil e na maior parte das grandes cidades da América Latina, prestase para presumir o possível impacto de uma intervenção específica - o emprego do ferro medicamentoso para o combate às anemias em condições marcadas pelo atraso econômico e social. Esta perspectiva, portanto, representaria um elemento prévio de validação do experimento como estratégia de saúde coletiva.

Sem dúvida, seria recomendável que, no diagnóstico inicial, tivessem sido adotados os mesmos procedimentos laboratoriais seguidos na avaliação seqüencial dos dois esquemas de tratamento. Isto, no entanto, implicaria em custos bem mais elevados e numa maior demora na produção dos resultados, extrapolando o cronograma estabelecido para o curso de experiência.

Por outro lado, as técnicas de diagnóstico tipo screening são plenamente aceitas e recomendáveis em estudos de campo, especialmente quando, em desenhos experimentais, se trabalha com o método de randomização (Mausner \& Bahn, 1990; Rouquayrol, 1994). As análises comparativas efetuadas asseguram que o processo de aleatorização possibilitou a formação de grupos homogêneos em relação às variáveis de interesse e, muito particularmente, em relação à variável resposta, ou seja, os níveis de hemoglobina.

As perdas registradas ao longo do processo experimental foram comparáveis entre os dois grupos. Tais perdas tinham as mesmas características do perfil original desses grupos, permitindo concluir que o processo de randomização e a seqüência de eventos ocorridos nos três meses das intervenções não produziram interferência ou viés nos resultados. Dispõe-se, por- tanto, da necessária consistência metodológica para validação do ensaio.

$\mathrm{O}$ fato de que $48 \%$ das mulheres que receberam o tratamento alternativo tenham normalizado seus níveis de hemoglobina, associado ao registro de que, após 12 semanas, nenhum caso tenha permanecido com menos de $9 \mathrm{~g} / \mathrm{dl}$ de hemoglobina, pode ser assumido como um bom resultado. Duas razões, pelo menos, justificam este juízo. Em primeiro lugar, pela referência de que a reversão do problema está acima da expectativa de 33\% (um terço), estabelecida pelas Nações Unidas no Plano de Metas para o ano 2000 (Encontro Mundial de Cúpula pela Criança, 1990) em relação à prevalência da anemia em mulheres no período reprodutivo. O segundo aspecto positivo referese à observação de que o percentual de cura situa-se um pouco acima dos resultados obtidos por Marques (1992), também no Recife, tratando gestantes anêmicas com três esquemas terapêuticos distintos, um dos quais incluindo, além do uso diário do ferro, a administração de ácido fólico e de vitamina $\mathrm{B}_{12}$.

No tratamento convencional, a percentagem de cura foi de 36,6\%. Mesmo não sendo estatisticamente diferente dos resultados obtidos com o esquema alternativo (48\%), praticamente coincide com a meta mínima de $33 \%$, referenciada pelas Nações Unidas. Ademais, com a administração diária de sais de ferro utilizada neste esquema, três casos $(4,2 \%$ do grupo) permaneceram com valores de hemoglobina abaixo de 9,0 g/dl. Em outras palavras, a persistência de formas moderadas de anemia e a menor proporção de curas, ainda que não estatisticamente significativa, somam evidências favoráveis ao tratamento alternativo, incluindo a observação adicional de que o aumento de hemoglobina neste grupo $(1,31 \mathrm{~g} / \mathrm{dl})$ também está acima do aumento obtido no grupo convencional $(0,90 \mathrm{~g} / \mathrm{dl})$. São evidências que, pelo 
caráter convergente, tornam-se indicativas de respostas mais eficazes ao tratamento alternativo. O único parâmetro que poderia, aparentemente, favorecer o tratamento convencional seria a persistência de microcitose no final da observação terapêutica (convencional, 17,8\%; alternativo, 22,0\%), sem significado estatístico em termos comparativos.

Os resultados relativos à aceitação do tratamento indicam que no primeiro mês, ou seja, após 4 semanas de realização do ensaio terapêutico, 91,3\% das mulheres apresentaram ótima aderência ao tratamento alternativo, enquanto no grupo convencional o percentual de aceitação ótima foi de $77,6 \%$. Nos dois meses seguintes, as taxas de aceitação ótima ao tratamento experimental se mantiveram iguais ou superiores a $90,0 \%$, o que constitui, sem dúvida, um resultado muito satisfatório, enquanto no modelo convencional estes valores se fixaram em $83,5 \%$.

Expresso de outra forma, significa dizer que, no segundo e no terceiro mês de tratamento, apenas $10 \%$ das usuárias não manifestaram uma adesão ótima ao esquema semanal, enquanto, no tratamento diário, este percentual se elevou para $17 \%$. Embora estas diferenças não tenham sido estatisticamente significativas, a repetição dos resultados pode ser tomada como manifestação de uma tendência que favorece, consistentemente, o tratamento alternativo.

Sob o aspecto terapêutico, a diferença no primeiro mês pode ser um elemento muito promissor, desde que se sabe, por uma experiência farmacológica bem consolidada através dos tempos, que as respostas mais expressivas ao tratamento da anemia carencial, ferropriva ou não, ocorrem nas primeiras semanas, tornando-se progressivamente decrescentes depois do primeiro mês (Marques, 1992). Nestas circunstâncias, a melhor aceitação do tratamento nos primeiros trinta dias passa a ser um elemento crucial para o seu sucesso.

A discussão sobre este tópico não pode se esgotar nos limites das observações aqui analisadas. Convém aprofundar os dados informativos, incluindo os motivos que justificariam as variações de conduta face à sistemática do tratamento. Efeitos colaterais? Esquecimento? Pouca motivação? Respostas a estas questões seriam úteis para conceber procedimentos corretivos, embora se possa considerar que taxas de aceitação ótima iguais ou acima de $90 \%$, conseguidas com o tratamento alternativo, já possam ser consideradas como um sucesso.

Em resumo, o percentual de cura das anemias, próximo a $50 \%$ em três meses de utiliza- ção semanal de fármacos de ferro, os níveis ótimos e satisfatórios de aderência dos usuários, o baixíssimo custo ( $14,3 \%$ do convencional) e, provavelmente, a reduzida ocorrência de efeitos colaterais com a administração de compostos de ferro a cada sete dias são dados muitos promissores em relação às perspectivas de luta contra a anemia em mulheres no período reprodutivo.

No contexto em que se desenvolveu a experiência e na consideração simultânea dos vários aspectos revelados pelo ensaio terapêutico, pode-se concluir que o esquema alternativo é recomendável e preferencial, em comparação aos modelos clássicos difundidos na rotina dos serviços de saúde.

\section{Recomendações}

Recomenda-se, em tratamentos prolongados de anemia ferropriva em mulheres "menstruantes", substituir o modelo convencional de administração diária de sais de ferro pelo esquema alternativo, com o uso semanal de compostos de ferro.

Torna-se oportuna e relevante a necessidade de aprofundar estudos no sentido de esclarecer as taxas, ainda elevadas, de fracassos nos casos resistentes à terapia com sais de ferro, com a administração semanal ou diária de sulfato ferroso.

Seria pertinente e interessante estudar, mediante desenhos de pesquisa especificamente concebidos para tal fim, as razões da não-adesão ao tratamento de anemia com sulfato ferroso.

Face à suposição de que sais de ferro administrados em doses menores poderiam diminuir os efeitos colaterais do tratamento, recomenda-se a realização de estudos para a validação ou não dessa hipótese.

Sabendo-se que mulheres em idade reprodutiva estão incluídas nos grupos de risco para a anemia e que, com certa freqüência, iniciam a gestação com reservas insuficientes de ferro, torna-se importante o desenvolvimento de um maior número de estudos e pesquisas sobre anemias no grupo de mulheres em idade fértil.

\section{Conclusões}

O percentual de cura de anemia em mulheres que menstruavam regularmente, com a administração semanal de sulfato ferroso, foi de $48,1 \%$ no tratamento alternativo, enquanto no 
tratamento convencional (uso diário de sulfato ferroso) o índice de cura alcançou 36,6\%.

Nos casos de anemia resistente ao tratamento convencional, após 12 semanas de uso de ferro medicamentoso, $17,8 \%$ eram do tipo microcítico. No fim do tratamento experimental, 22,0\% dos casos de anemia que não responderam ao tratamento foram classificados como microcitose.

No primeiro mês de experiência, as taxas de adesão ao tratamento alternativo (91,3\% consi- deradas ótimas) foram, estatisticamente, mais elevadas que no modelo convencional $(77,6 \%$ ótima). Nos meses seguintes, os percentuais de aceitação aos dois esquemas de tratamento foram equivalentes.

Na avaliação conjunta dos vários resultados pode-se concluir que o tratamento semanal dos casos de anemia em mulheres "menstruantes” é mais vantajoso em relação ao esquema convencional do uso diário de sais de ferro.

\section{Referências}

ADISH, A. \& ESREY, S. A., 1996. Deficiência de ferro e anemia: Um premente problema mundial. A Prescripção, 11:16.

ARRUDA, I. K. G., 1990. Prevalência de Anemia em Gestantes de Baixa Renda: Algumas Variáveis Associadas e sua Repercussão no Recém-Nascido. Dissertação de Mestrado, Recife: Departamento de Nutrição, Universidade Federal de Pernambuco.

ARRUDA, I. K. G., 1997. Deficiência de Ferro, de Folato e Anemia em Gestantes Atendidas no Instituto Materno Infantil de Pernambuco: Magnitude, Fatores de Risco e Algumas Implicações nos seus Conceptos. Tese de Doutorado, Recife: Departamento de Nutrição, Universidade Federal de Pernambuco.

BATISTA FILHO, M. \& FERREIRA, L. O. C., 1996. Prevenção e tratamento da anemia nutricional ferropriva: Novos enfoques e perspectivas. Cadernos de Saúde Pública,12:37-41.

CARDOSO, M. A. \& PENTEADO, M. V. C., 1994. Intervenções nutricionais na anemia ferropriva. $\mathrm{Ca}$ dernos de Saúde Pública, 10:231-240.

DALLMAN, P. R., 1991. Hierro. Revista de la Organización Panamericana de la Salud. (Publicación Científica 532) pp. 277-288, Washington, D. C.: Organización Panamericana de la Salud/Instituto Internacional de Ciencias de la Vida.
ENCONTRO MUNDIAL DE CÚPULA PELA CRIANÇA, 1990. Declaração Mundial sobre a Sobrevivência, a Proteção e o Desenvolvimento da Criança e Plano de Ação para a Implementação da Declaração Mundial sobre a Sobrevivência, a Proteção e o Desenvolvimento da Criança nos Anos 90. Brasília: UNICEF (United Nations Children's Fund).

FINCH, C. A. \& COOK, J. D., 1984. Iron deficiency. American Journal of Clinical Nutrition, 39:471477.

HERCBERG, S. \& GALAN, P., 1992. Nutritional anaemias. Baillieres Clinical Haematology, 5:143-164.

HILLMAN, R. S., 1991. Agentes hematopoiéticos: Fatores de crescimento, sais minerais e vitaminas. In: Goodman \& Gilman. As Bases Farmacológicas da Terapêutica (A. G. Gilman, T. W. Rall, A. S. Nies \& P. Taylor, eds.), 8a Ed., pp. 851-873, Rio de Janeiro: Guanabara Koogan.

IBGE (Fundação Instituto Brasileiro de Geografia e Estatística), 1992. Anuário Estatístico do Brasil, Resenha Histórica. Rio de Janeiro: Secretaria de Planejamento, Orçamento e Coordenação.

IBGE (Fundação Instituto Brasileiro de Geografia e Estatística), 1996. A Contagem Populacional de 1996. Dados Preliminares. Recife: IBGE.

KIRKWOOD, B. R., 1988. Essentials of Medical Statistics. London: Blackwell. 
KJELDSBERG, C. R., 1993. Principles of hematologic examinations. In: Wintrobe's Clinical Hematology (G. R. Lee, T. C. Bithell, J. Foerster, J. W. Athens \& J. N. Lukens, eds.), 9. Ed., pp. 101-133, London: Lea \& Febinger.

LIPKIN, M.; SHERLOCK, P. \& BELL, B., 1963. Cell proliferation kinetics in the gastrointestinal tract of man. Gastroenterology, 45:721-729.

MARQUES, A. P. O., 1992. Avaliação de Três Esquemas de Intervenção em Gestantes Anêmicas. Dissertação de Mestrado, Recife: Departamento de Nutrição, Universidade Federal de Pernambuco.

MARTINS, I. S.; ALVARENGA, A. T.; SIQUEIRA, A. A. R. \& LIMA, B. D., 1987. As determinações biológicas e sociais da doença: Um estudo da anemia ferropriva. Revista de Saúde Pública, 21:73-89.

MAUSNER, J.S. \& BAHN, A. K. 1990. Ensaios profiláticos e terapêuticos: Estudos experimentais. In: Introdução à Epidemiologia (J. S. Mausner \& A. K. Bahn, org.), pp. 293-306, Lisboa: Fundação Calouste Gulbenkian.

NACIONES UNIDAS, 1987. First Report on the World Nutrition Situation. Roma: Comité Administrativo de Coordinación, Subcomité de Nutrición.

OLIVEIRA, I., 1990. Anemia por deficiência de ferro. In: Pediatria (F. Figueira, O. S. Ferreira \& J. O. B. Alves, org.), pp. 543-548, Rio de Janeiro: MEDSI.

PAHO/WHO (Pan American Health Organization/ World Health Organization), 1994. Nutritional situation in the anemias. Epidemiological Bulletin, 15:1-6.

PEREIRA, R. C., 1997. Anemia em Parturientes da Maternidade Professor Monteiro de Moráes e Peso ao Nascer: Impacto de Condicionantes Macro e Microestruturais. Dissertação de Mestrado, Recife: Departamento de Nutrição, Universidade Federal de Pernambuco.
PREFEITURA DA CIDADE DO RECIFE, 1993. Situação Econômica e Social da Cidade do Recife. Recife: Departamento de Informações e Projeções, Diretoria Geral de Desenvolvimento Urbano e Ambiental, Secretaria de Planejamento Urbano e Ambiental.

RAPAPORT, S. I., 1990. Introdução à Hematologia. São Paulo: Rocca.

ROUQUAYROL, M. Z., 1994. Epidemiologia \& Saúde. Rio de Janeiro: MEDSI.

RYBO, G., 1970. Menstrual loss of iron. In: Iron Deficiency. Pathogenesis. Clinical Aspects. Therapy. (L. Halberg, ed.), pp. 163-171, London: Academic Press.

SALZANO, A. C.; BATISTA-FILHO, M. \& FLORES, H., 1980. Prevalência de anemia no ciclo gestacional, em dois Estados do Nordeste brasileiro, Pernambuco e Paraíba. Revista Brasileira de Pesquisas Médicas e Biológicas, 13:211-214.

SCHULTINK, W.; RAINER, G.; GLIWINTTZKI, M.; KARYADI, D. \& MATULESSI, P., 1995. Effect of daily vs twice weekly iron supplementation in Indonesian preschool children with low iron status. American Journal of Clinical Nutrition, 61:111115.

STEKEL, A. C., 1984. Prevention of iron deficiency. In: Iron Nutrition in Infancy and Childhood (A. Stekel, ed.), pp. 179-194, Nestlé Nutrition Workshop Series, New York: Raven Press.

VITERI, F. E.; HERCBERG, S.; GALAN, P.; GUIRO, A. \& PREZIOSI, P., 1993. Absortion of iron supplements administred daily or weekly: A colaborative study. In: Nestlé Fundation for the Study of the Problems of Nutrition in the World. Annual Report, pp. 82-96, Genève: Nestlé. 